\title{
Educating caregivers to recognise the clinical signs of pneumonia in children younger than 6 months
}

\author{
D Ansong, ${ }^{1,2,4} \mathrm{MD}$, MSc; F A Osei, ${ }^{3} \mathrm{MPH}$; S K Owusu ${ }^{2} \mathrm{MD}$; I Nyanor, ${ }^{4} \mathrm{MPH}$; J Bonney, ${ }^{4} \mathrm{MD}, \mathrm{MPH}$; A Enimil, ${ }^{2} \mathrm{MD}$; \\ D O Laryea, ${ }^{3} \mathrm{MD}, \mathrm{MSc}$; J Dapaah, ${ }^{5} \mathrm{PhD}$; P Agyei-Baffour, ${ }^{6} \mathrm{PhD} ; \mathbf{N}$ Mensah, ${ }^{1} \mathrm{BSc}$; Osei-Peprah, ${ }^{1} \mathrm{BSc} ;$ A Owusu, ${ }^{1} \mathrm{BSc}$; \\ E Addo-Yobo, ${ }^{1,2} \mathrm{MD}$, DM; A Osei-Akoto, ${ }^{1,2} \mathrm{MD}$; O A Owusu, ${ }^{4} \mathrm{MD}$; S C Y Appiah, ${ }^{5}$ MPhil; V Ampiah, ${ }^{5} \mathrm{MPhil}$; J O Saahene, ${ }^{4} \mathrm{MPH}$; \\ E X Amuzu, ${ }^{4}$ BSc; M Telly, ${ }^{3}$ BSc; J Sylverken, ${ }^{2}$ MD \\ ${ }^{1}$ School of Medical Sciences, Kwame Nkrumah University of Science and Technology, Kumasi, Ghana \\ 2 Department of Child Health, Komfo Anokye Teaching Hospital, Kumasi, Ghana \\ ${ }^{3}$ Public Health Unit, Komfo Anokye Teaching Hospital, Kumasi, Ghana \\ ${ }^{4}$ Research and Development Unit, Komfo Anokye Teaching Hospital, Kumasi, Ghana \\ ${ }^{5}$ Department of Sociology and Social Work, Kwame Nkrumah University of Science and Technology, Kumasi, Ghana \\ ${ }^{6}$ School of Public Health, Kwame Nkrumah University of Science and Technology, Kumasi, Ghana
}

Corresponding author: F A Osei (francisph1@hotmail.com)

\begin{abstract}
Background. Pneumonia remains the foremost cause of death in young children in sub-Saharan Africa. This phenomenon is largely driven by poor access to healthcare and delay in seeking medical care for childhood pneumonia.

Objective. To assess the effectiveness of training caregivers to recognise the early clinical signs of pneumonia.

Methods. The study involved a cohort of women presenting to the Child Welfare Clinic at the Komfo Anokye Teaching Hospital in Kumasi, Ghana, between 7 July and 8 September 2016. A total of 90 women with children younger than 10 weeks were recruited. Participants were trained on identifying early signs of pneumonia using low-cost equipment. Follow-up training and assessment sessions formed part of the programme.

Results. At pre-training assessment, the majority of the participants $(n=83 / 90 ; 92.2 \%)$ recognised lower chest indrawing as a sign of respiratory disease requiring immediate hospital intervention. Participants' performance in determining rhythms of 50 breaths per minute $(\mathrm{bpm})$ and $60 \mathrm{bpm}$ improved significantly across sessions ( $p=0.011$ and $p \leq 0.001$, respectively). After training, 87 participants ( $96.7 \%)$ were able to determine rapid breathing accurately compared with 73 participants $(81.1 \%)$ before training $(p=0.001)$.

Conclusion. The results suggest that caregivers can be effectively trained to identify clinical signs of pneumonia in young children, even in low-resource settings. A training initiative as described in this study could be an effective public health intervention to help address the burden of pneumonia in low-resource settings.
\end{abstract}

S Afr J Child Health 2020;14(2):70-76. https://doi.org/10.7196/SAJCH.2020.v14i2.1642

Pneumonia remains a significant contributor to preventable deaths in children under five. ${ }^{[1]}$ Together, pneumonia and diarrhoea account for $30 \%$ of all deaths in this age group, which translates to a loss of two million lives each year. ${ }^{[2]}$ The global death toll of pneumonia was estimated at 1.3 million in 2011 , with $81 \%$ of fatalities occurring in the first two years of life. ${ }^{[3]}$

More than 150 million cases of pneumonia are reported in lowand-middle-income countries per year. ${ }^{[4]}$ In sub-Saharan Africa, it is the leading cause of mortality in children under five and accounts for 490000 deaths annually. ${ }^{[5]}$

Children living in low socioeconomic households or remote areas are the worst affected. In many cases, these children report to facilities only at a late stage and may have complicated pneumonia episodes, subsequently associated with poor outcomes. The burden of pneumonia on the families and general health systems in lowresource settings further worsens the existing health inequities, as pneumonia diagnosed late is more expensive to treat owing to complications. ${ }^{[2]}$

Considerable gains have been made in reducing morbidity and mortality from pneumonia following the introduction of vaccines against Haemophilus influenzae, pneumococcal disease, pertussis and measles as part of the Expanded Programme on Immunisation in Ghana. ${ }^{[6,7]}$ Implementing treatment protocols as included in the
Integrated Management of Neonatal and Childhood Illness approach has resulted in further progress. Currently, pneumonia morbidity and mortality seem to be influenced largely by delays in diagnosis and initiation of treatment. ${ }^{[8,9]}$

The World Health Organization has documented more than ten simple non-technological interventions to promote child survival and to reduce mortality from pneumonia. ${ }^{[2]}$ However, these interventions focus mostly on healthcare workers, health facilities and access to essential medicines such as antibiotics, and do not help caregivers at home to detect diseases such as pneumonia early.

A study of children with pneumonia, as documented by the receiving doctor at a health facility in New Delhi, India, reported that $\sim 80 \%$ of mothers $(n=161 / 200)$ identified difficulty in breathing as the most distressing sign for which they sought medical attention, despite their not previously being trained on recognising signs of pneumonia. ${ }^{[10]}$ Although difficulty in breathing is a late sign of pneumonia, this finding suggests that educating mothers on identifying early signs of pneumonia may facilitate early case detection and treatment to avert complications and death. ${ }^{[10]}$

Strategies to further reduce the burden of pneumonia in lowincome countries need not require advanced technologies. ${ }^{[2]}$ During episodes of pneumonia, rapid breathing is a key symptom and an independent predictor for hospital death. ${ }^{[11]}$ Educating caregivers, 
especially mothers, to recognise early signs of pneumonia and seek care promptly may be a crucial step in reducing avoidable pneumonia morbidity and mortality. ${ }^{[10]}$

Educating caregivers to detect early signs of pneumonia remains an unexplored opportunity for early diagnosis and prompt treatment to reduce the medical and social burden of the disease. This study therefore set out to assess the effectiveness of training mothers and primary caregivers to recognise the early clinical signs of pneumonia.

\section{Methods}

\section{Study design and inclusion criteria}

The study involved a cohort of women with children younger than 10 weeks who presented to the Child Welfare Clinic at the Komfo Anokye Teaching Hospital (KATH) for vaccination and growth monitoring between 7 July and 8 September 2016. The women's ability to determine respiratory rate and detect respiratory problems in their infants before and after training was evaluated. Participants had to be willing to complete the full training period to be eligible for inclusion in the study. Mothers with children older than 10 weeks were excluded from the study.

\section{Setting}

The KATH is located in Kumasi, the regional capital of Ashanti Region, Ghana, with an estimated total population of 4780380 based on 2010 census data. ${ }^{[12]}$ This 1200 bed hospital is a tertiary healthcare facility (referral centre) and a teaching hospital. The Public Health Unit administers immunisations for pregnant women and children up to 5 years at the clinic, which operates from Monday to Friday.

\section{Sampling}

The required sample size was calculated as $N=90$, based on a $95 \%$ confidence interval and a power of $80 \%$ to detect an intraobserver rate of $20 \%$ for before and after training assessment. This intraobserver rate was estimated based on a study conducted among Ugandan community health workers to evaluate their ability to assess rapid breathing in under-fives. The authors reported a $71 \%$ accuracy rate, with cases correctly identified to within 5 breaths relative to a baseline count used as standard. ${ }^{[9]}$

The women were approached for participation at the clinic during operating hours each day. Of the 111 eligible mothers who were approached, 90 agreed to participate (Fig. 1). The main reason for refusal to participate was that the candidates could not commit their availability over the full study period.

\section{Study procedure \\ Training sessions}

Participants attended three training sessions in small groups during the course of the study. The teaching instruments included an hourglass (as respiratory timer), coloured beads (for counting breaths), a television set, structured video presentations and flash cards. Training was conducted by a team of clinicians from the Department of Child Health and the Public Health Unit at KATH. Each training session consisted of (i) recognising early signs of pneumonia, (ii) using coloured beads in determining and interpreting respiratory rates, and (iii) recognising danger signs of pneumonia. Practical sessions during which participants demonstrated the techniques were included to ensure that the participants were competent in determining respiratory rate. The training sessions were standardised and piloted before recruitment of study participants.

\section{Follow-up sessions}

Participants attended refresher training and assessment at the hospital twice (at 4 and 8 weeks after the initial training, respectively) (Fig. 1). Assessment involved counting simulated breaths accurately, real-case counting and correctly recognising other clinical signs of pneumonia. The refresher training and follow-up assessment occurred on the same day. Participants received three scheduled follow-up phone calls to remind them of their next visit and encourage them to practise the counting skills they had learnt in preparation for the post-training evaluation at a subsequent scheduled clinic visit.

\section{Data collection and analysis}

A modular approach was used to assess participants' ability to determine respiratory rate and detect other signs of pneumonia. During the first module, participants were asked to use the coloured beads and timers to determine the rate of beeps (sounds) presented at 32, 50 and 60 breaths per minute (bpm); the beeps simulated breaths. The second module required participants to determine respiratory rate from audiovisual material while using the beads and timers. The respiratory rates reported by each participant were recorded. During the final assessment module, participants were required to identify various clinical signs of pneumonia from videos.

Participants were also taught to detect various signs of upper and lower respiratory tract diseases. Signs of upper respiratory tract disease included cough, rhinorrhoea and stridor, whereas signs of lower respiratory tract disease included nasal flaring, grunting, head bobbing, indrawing of the lower chest wall, intercostal recession and cyanosis.

Data were captured electronically using the Open Data Kit system and analysed using STATA/MP 13.0 (StataCorp., USA). Descriptive statistics were used for both

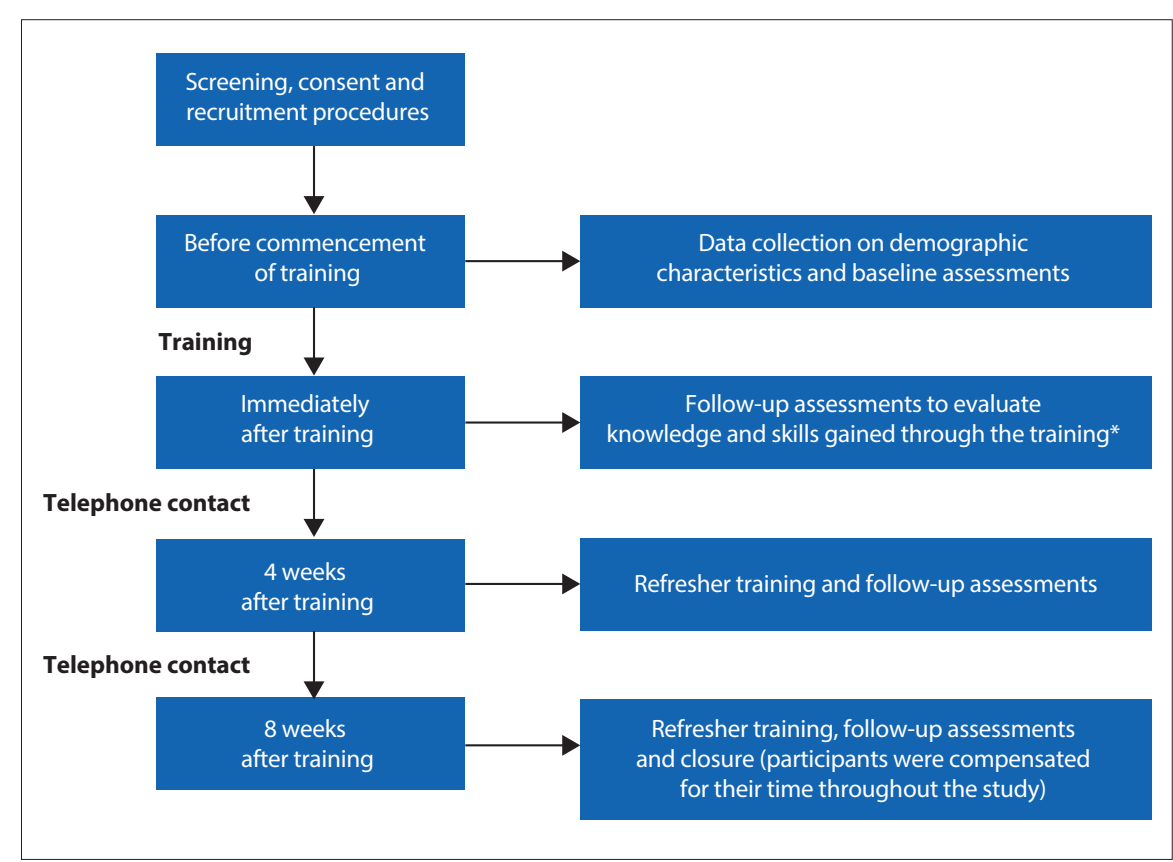

Fig. 1. Workflow and follow-up assessments. $\left({ }^{*}\right.$ This can be attributed to consistent participant contact and scheduling follow-up sessions to coincide with participants' confirmed clinic visits.) 
continuous and categorical variables. Categorical variables included participants' characteristics (age, educational background and occupation) and knowledge of the clinical symptoms of respiratory tract diseases, including pneumonia.

Respiratory rates reported to within 1 count of the actual rate were recorded as correct during the simulations. As participants were shown a video of a normal respiratory rate in the second assessment module, reported rates that were within the normal respiratory rate were judged to be correct. Similarly, breathing rates correctly identified as abnormal when participants viewed videos of increased breathing rates were recorded as a correct result. Success in the third module involved participants' correctly identifying normal and abnormal respiratory signs from videos.

The rate of accuracy was determined by comparing participants' reported rates with the actual rates. The change in accuracy over the 8 -week period was determined by means of a $\chi 2$ test.

\section{Ethical considerations}

Permission to conduct the study was obtained from the Committee on Human Research Publications and Ethics, a joint committee of the School of Medical Sciences at the Kwame Nkrumah University of Science and Technology and KATH (ref. no. CHRPE/AP/384/16).

The purpose of the study, the risks and benefits of participation, and the study procedures were explained to prospective participants, along with providing information about the investigators, collaborating institutions and the funding agency. Participants were allowed to complete the usual growth monitoring and immunisation routines before presenting for the training and assessment sessions. All participants gave written informed consent to confirm their willingness to participate.

\section{Results}

A total of 90 participants were recruited for the study. The mean age (and associated standard deviation (SD)) of participants was 30.9 (5.4) years. Approximately two-thirds (67.8\%) of the participants had completed at least senior secondary schooling. The majority of the participants $(80.0 \%)$ were employed and married (81.1\%) (Table 1).

Participants' knowledge of clinical signs of pneumonia before and after training is summarised in Table 2. Before training, cough lasting up to 2 weeks was identified as a symptom of respiratory disease most often $(52.2 \%)$. The least familiar sign of respiratory disease was head bobbing, which was identified by only $21.1 \%$ of participants. A sustained increase in recall of clinical signs of pneumonia and other respiratory tract diseases was seen after training (Table 2).

Before training, the majority of participants (92.2\%) recognised lower chest indrawing as a sign of abnormal breathing, whereas intercostal recession was recognised as a sign of abnormal breathing least often (Table 3). Improved recognition was observed during assessment immediately after training, as none of the participants indicated intercostal recession and lower chest indrawing as normal signs. In subsequent sessions, participants were able to identify accurately the signs of normal and abnormal breathing and so predict danger signs of pneumonia. For example, at both 4 and 8 weeks after training, all participants identified nasal flaring as a sign of abnormal breathing and which requires immediate hospital intervention. Similarly, all but one of the participants $(98.9 \%)$ identified intercostal recession as a sign of abnormal breathing.

Participants' ability to determine and interpret various simulated breathing rates using coloured beads was compared with that of a clinician's counting. The results showed that $63.3 \%-75.6 \%$ of participants were able to determine the various respiratory rates accurately before training (Table 4). A marginal increase in performance across the different rates $(77.8 \%-81.1 \%)$ was observed 4 weeks after training, following continued practice with the counting device at home. Assessment after 8 weeks showed a further improvement in performance among participants $(84.4 \%-86.7 \%)$.

Table 5 summarises participants' assessments of respiratory rate from video recordings after being trained in rhythmic counting using coloured beads and timers. Results showed that most participants were able to assess breathing rate correctly already in the first session, immediately after training with beeps. Similar performance levels were observed at exit training. A notable change in performance levels was observed during session 2, with accuracy rates ranging between $78.9 \%$ and $83.3 \%$ across cases. However, performance

\begin{tabular}{ll} 
Table 1. Participant characteristics $(\mathbf{N = 9 0 )}$ & \\
\hline Characteristic & $\boldsymbol{n}(\mathbf{\%})$ \\
\hline Age (years) & \\
$<30$ & $39(43.3)$ \\
$30-35$ & $30(33.3)$ \\
$>35$ & $21(23.3)$ \\
Education status & \\
No formal education & $6(6.7)$ \\
Basic & $23(25.5)$ \\
Senior secondary school & $33(36.7)$ \\
Vocational/technical/polytechnical qualification & $9(10.0)$ \\
University degree & $19(21.1)$ \\
Employment status & \\
Unemployed & $9(10.0)$ \\
Menial work & $22(24.4)$ \\
Civil servant & $32(35.6)$ \\
Trader & $27(30.0)$ \\
Marital status & \\
Single/never married & $7(7.8)$ \\
Married & $73(81.1)$ \\
Cohabiting & $10(11.1)$ \\
& $*$ Primary and junior secondary school. \\
&
\end{tabular}

Table 2. Recognition of symptoms of respiratory tract disease, including pneumonia, before and after training $(N=90)$

\begin{tabular}{llll}
\hline & $\begin{array}{l}\text { Before } \\
\text { training, }\end{array}$ & \multicolumn{3}{c}{ After training } \\
\cline { 3 - 4 } Symptom & $\begin{array}{l}\text { After 4 weeks, } \\
n(\%)\end{array}$ & $\begin{array}{l}\text { After 8 weeks, } \\
n(\%)\end{array}$ \\
\hline Fever & $22(24.4)$ & $72(80.0)$ & $83(92.2)$ \\
Fast breathing & $35(38.9)$ & $87(96.7)$ & $86(95.6)$ \\
Poor feeding & $26(28.9)$ & $84(93.3)$ & $82(91.1)$ \\
Cough lasting & $35(38.9)$ & $73(81.1)$ & $71(78.9)$ \\
$\begin{array}{l}3 \text { days } \\
\text { Cough lasting }\end{array}$ & $47(52.2)$ & $85(94.4)$ & $87(96.7)$ \\
1 - 2 weeks & & & \\
Runny nose & $25(27.8)$ & $71(78.9)$ & $71(78.9)$ \\
Grunting & $43(47.8)$ & $86(95.6)$ & $89(98.9)$ \\
Head bobbing & $19(21.1)$ & $84(93.3)$ & $82(91.1)$ \\
Stridor & $49(54.4)$ & $81(90.0)$ & $86(95.6)$ \\
${ }^{*}$ As a sign of pneumonia. & & &
\end{tabular}


Table 3. Participants' interpretation of other clinical signs of respiratory tract diseases $(N=90)$

\begin{tabular}{|c|c|c|c|}
\hline Signs & $\begin{array}{l}\text { Normal } \\
\text { breathing, } \\
n(\%)\end{array}$ & $\begin{array}{l}\text { Abnormal } \\
\text { breathing, } \\
n(\%)\end{array}$ & $\begin{array}{l}\text { Do not } \\
\text { know, } \\
n(\%)\end{array}$ \\
\hline \multicolumn{4}{|l|}{ Before training } \\
\hline Nasal flaring & $4(4.4)$ & $75(83.3)$ & $11(12.2)$ \\
\hline No flaring & $76(84.4)$ & $8(8.89)$ & $6(6.7)$ \\
\hline Intercostal recession & $2(2.2)$ & $81(90.0)$ & $7(7.8)$ \\
\hline $\begin{array}{l}\text { No intercostal } \\
\text { recession }\end{array}$ & $74(82.2)$ & $12(13.3)$ & $4(4.4)$ \\
\hline Cyanosis & $4(4.4)$ & $74(82.2)$ & $12(13.3)$ \\
\hline Lower chest indrawing & $4(4.4)$ & $83(92.2)$ & $3(3.3)$ \\
\hline \multicolumn{4}{|l|}{ Immediately after training } \\
\hline Nasal flaring & $3(3.3)$ & $86(95.6)$ & $1(1.1)$ \\
\hline $\begin{array}{l}\text { Normal face with no } \\
\text { flaring }\end{array}$ & $84(93.3)$ & $4(4.4)$ & $2(2.2)$ \\
\hline Intercostal recession & $0(0)$ & $88(97.8)$ & $2(2.2)$ \\
\hline $\begin{array}{l}\text { Normal chest movement } \\
\text { with no intercostal }\end{array}$ & $81(90.0)$ & $8(8.9)$ & $1(1.1)$ \\
\hline Cyanosis & $1(1.1)$ & $88(97.8)$ & $1(1.1)$ \\
\hline Lower chest indrawing & 0 & $89(98.9)$ & $1(1.1)$ \\
\hline \multicolumn{4}{|l|}{ At 4 weeks after training } \\
\hline Nasal flaring & 0 & $90(100)$ & 0 \\
\hline $\begin{array}{l}\text { Normal face with no } \\
\text { flaring }\end{array}$ & $87(96.7)$ & $3(3.3)$ & 0 \\
\hline Intercostal recession & $1(1.1)$ & 89 (98.9) & 0 \\
\hline $\begin{array}{l}\text { Normal chest movement } \\
\text { with no intercostal }\end{array}$ & $88(97.8)$ & $1(1.1)$ & $1(1.1)$ \\
\hline Cyanosis & $2(2.2)$ & $88(97.8)$ & 0 \\
\hline Lower chest indrawing & $2(2.2)$ & $88(97.8)$ & 0 \\
\hline \multicolumn{4}{|l|}{ At 8 weeks after training } \\
\hline Nasal flaring & 0 & $90(100)$ & 0 \\
\hline $\begin{array}{l}\text { Normal face with no } \\
\text { flaring }\end{array}$ & $86(95.6)$ & $3(3.3)$ & $1(1.1)$ \\
\hline Intercostal recession & 0 & 89 (98.9) & $1(1.1)$ \\
\hline $\begin{array}{l}\text { Normal chest movement } \\
\text { with no intercostal }\end{array}$ & $85(94.4)$ & $5(5.6)$ & 0 \\
\hline Cyanosis & $3(3.3)$ & $86(95.6)$ & $1(1.1)$ \\
\hline Lower chest indrawing & $1(1.1)$ & 89 (98.9) & 0 \\
\hline
\end{tabular}

levels increased again by session 3 ( 8 weeks after initial training). For example, 73 participants $(81.1 \%)$ correctly reported respiratory rate during rapid breathing in session 2, whereas 87 participants $(96.7 \%)$ were able to report respiratory rate accurately for this case by session 3 ( $p=0.001)$.

\section{Discussion}

The study has shown that caregivers can be effectively trained to determine respiratory rate and identify symptoms of respiratory disease in young infants, regardless of educational and socioeconomic status. A caregiver's ability to identify early signs of pneumonia at home can contribute to patients' presenting to a health facility in time for prompt treatment and also help to reduce the morbidity and mortality burden associated with late treatment. The results showed a significant change in knowledge and skill levels after training, sustained by intermittent refresher training. We argue that participants in such programmes will be able to retain and practise
Table 4. Participants' performance on counting beeps simulating rhythmic breathing $(N=90)$

\begin{tabular}{|c|c|c|c|}
\hline Assessment stage & $\begin{array}{l}\text { Correct } \\
\text { count, } n(\%)\end{array}$ & $\begin{array}{l}\text { Incorrect } \\
\text { count, } n(\%)\end{array}$ & $p$-value \\
\hline \multicolumn{4}{|c|}{ Rhythmic counting: $32 \mathrm{bpm}$} \\
\hline Before training & $68(75.6)$ & $22(24.4)$ & 0.199 \\
\hline $\begin{array}{l}\text { Immediately after } \\
\text { training }\end{array}$ & $72(80.0)$ & $18(20.0)$ & \\
\hline 4 weeks after training & $70(77.8)$ & $20(22.2)$ & \\
\hline 8 weeks after training & $76(84.4)$ & $14(15.6)$ & \\
\hline \multicolumn{4}{|c|}{ Rhythmic counting: 50 bpm } \\
\hline Before training & $62(68.9)$ & $28(31.1)$ & $0.011^{*}$ \\
\hline $\begin{array}{l}\text { Immediately after } \\
\text { training }\end{array}$ & $72(80.0)$ & $18(20.0)$ & \\
\hline 4 weeks after training & $74(82.2)$ & $16(17.8)$ & \\
\hline 8 weeks after training & $76(84.4)$ & $14(15.6)$ & \\
\hline \multicolumn{4}{|c|}{ Rhythmic counting: $60 \mathrm{bpm}$} \\
\hline Before training & $57(63.3)$ & $33(36.7)$ & $<0.001^{\star}$ \\
\hline $\begin{array}{l}\text { Immediately after } \\
\text { training }\end{array}$ & $70(77.8)$ & $20(22.2)$ & \\
\hline 4 weeks after training & $73(81.1)$ & $17(18.9)$ & \\
\hline 8 weeks after training & $78(86.7)$ & $12(13.3)$ & \\
\hline \multicolumn{4}{|c|}{$\begin{array}{l}\text { bpm }=\text { breaths per minute. } \\
{ }^{*} \text { Statistically significant }(p<0.05) \text { according to } \chi^{2} \text { test of association. }\end{array}$} \\
\hline
\end{tabular}

their skills with regular training. The successful attainment of competencies in recognising the signs of respiratory disease may be attributed, in part, to the modular and graded training (first teaching with the beeps, then videos and real cases), but also to repeating the training over time to reinforce the learning.

An increased respiratory rate combined with signs such as cough and fever are well-documented clinical signs of pneumonia. ${ }^{[10,13,14]}$ However, little published research is available on training caregivers to determine respiratory rate. The present study puts forward a possible approach to and process for training caregivers effectively.

Determining respiratory rate should not be seen as a task exclusive to health professionals; rather, any caregiver who is willing to learn the skill should have access to training. The main challenge in accurately determining respiratory rate relates to mastering the rhythmic character of counting, and keeping the pace despite occasional irregularities. It was reassuring to find that a graded approach and repeated follow-up sessions yielded increasing accuracy. Learning how to determine respiratory rate accurately during this training and what danger signs to be aware of can help mothers to assess the wellbeing of their infant. The approach outlined in this study could well form part of a preventive and early detection process in the primary healthcare setting.

Recognising the clinical signs of pneumonia requires a caregiver to be aware of warning signs such as an increased respiratory rate, nasal flaring, intercostal or subcostal recession, lower chest indrawing and cyanosis. Although all of these are clinical signs of respiratory infections, increased respiratory rate is considered an early clinical warning sign, ${ }^{[11]}$ and hence our approach focused on training caregivers to recognise rapid breathing. In a study to assess mothers' recognition of signs of pneumonia in India, few participants were aware of rapid breathing being an early warning sign and limited knowledge of other early signs was also reported; however, refusal to feed was identified as a warning sign by almost all participants. ${ }^{[10]}$ Our study yielded similar 
Table 5. Participants' performance when determining an infant's respiratory rate from video recordings, using a clinician's assessment as standard $(\mathrm{N}=90)$

\begin{tabular}{|c|c|c|c|c|c|c|c|c|c|}
\hline \multirow[b]{2}{*}{ Video recording } & \multicolumn{2}{|c|}{$\begin{array}{l}\text { Session } 1 \text { (immediately } \\
\text { post rhythmic counting) }\end{array}$} & \multicolumn{2}{|c|}{$\begin{array}{l}\text { Session } 1 \text { (post case } \\
\text { training assessment) }\end{array}$} & \multicolumn{2}{|c|}{$\begin{array}{c}\text { Session } 2 \\
\text { (4 weeks post training) }\end{array}$} & \multicolumn{2}{|c|}{$\begin{array}{c}\text { Session } 3 \\
\text { (8 weeks post training) }\end{array}$} & \multirow[b]{2}{*}{$p$-value } \\
\hline & $\begin{array}{l}\text { Correct, } \\
n(\%)\end{array}$ & $\begin{array}{l}\text { Incorrect, } \\
n(\%)\end{array}$ & $\begin{array}{l}\text { Correct, } \\
n(\%)\end{array}$ & $\begin{array}{l}\text { Incorrect, } \\
n(\%)\end{array}$ & $\begin{array}{l}\text { Correct, } \\
n(\%)\end{array}$ & $\begin{array}{l}\text { Incorrect, } \\
n(\%)\end{array}$ & $\begin{array}{l}\text { Correct, } \\
n(\%)\end{array}$ & $\begin{array}{l}\text { Incorrect, } \\
n(\%)\end{array}$ & \\
\hline Case 1: Normal BR & $87(96.7)$ & $3(3.3)$ & $84(93.3)$ & $6(6.7)$ & $71(78.9)$ & $19(21.1)$ & $80(88.9)$ & $10(11.1)$ & $0.009^{*}$ \\
\hline Case 2: Normal BR & $85(94.4)$ & $5(5.6)$ & $82(91.1)$ & $8(8.9)$ & $72(80.0)$ & $18(20.0)$ & $86(95.6)$ & $4(4.4)$ & 0.578 \\
\hline Case 3: Normal BR & $84(93.3)$ & $6(6.7)$ & $78(86.7)$ & $12(13.3)$ & $75(83.3)$ & $15(16.7)$ & $83(92.2)$ & $7(7.8)$ & 0.653 \\
\hline $\begin{array}{l}\text { Case 4: Abnormal } \\
\text { breathing rate }\end{array}$ & - & - & - & - & $73(81.1)$ & $17(18.9)$ & $87(96.7)$ & $3(3.3)$ & $0.001^{*}$ \\
\hline
\end{tabular}

observations; less than half of the participants knew that rapid breathing is a sign of pneumonia in children prior to the training.

Our findings suggest that repeated training is an effective approach to improving caregivers' ability to detect warning signs such as nasal flaring, intercostal recession and lower chest indrawing, as most were able to identify these signs correctly after training and improvements in performance were seen over time. This further points to the potential effectiveness of simple training initiatives to educate mothers on identifying early warning signs of pneumonia and encourage them to seek healthcare promptly.

\section{Study limitations}

The study did not measure whether a training intervention such as this will directly lead to reduced hospital admissions and mortality. However, we argue that improved early detection by parents, and subsequent early reporting, could translate to reduced morbidity and mortality. ${ }^{[8,9]}$ Further studies to assess the effect of training interventions on healthcare costs and outcomes such morbidity and mortality will be useful.

The purposive sampling technique could have led to selection bias. Observational bias and group effect are also possible. As training was delivered in a group setting, it is possible that group psychology could have influenced counting accuracy.

\section{Conclusion}

The study showed that training initiatives can equip mothers to determine respiratory rate accurately and identify clinical signs of pneumonia early, even in low-resource settings. The results are particularly relevant in areas with a high burden of childhood pneumonia and provides a potential public health intervention for improving access to appropriate care. Exploring the possible integration of a training initiative such as this one into public health education for mothers at antenatal, postnatal or immunisation clinics is recommended. Instituting this as a policy intervention in the existing continuum of care programmes (antenatal care, skilled birth attendance and postnatal care, which have proven to be effective in other studies $)^{[15-17]}$ can contribute to improving the health of infants and young children. Although the cost benefit of training has not been analysed, the low cost associated with a training initiative of this kind and the potential contribution to early detection and consequent reduction in costly hospital care or preventing deaths suggests training initiatives to be attractive primary healthcare interventions.
Declaration. None.

Acknowledgements. We thank the healthcare workers and participants who agreed to take part in this study.

Author contributions. DA, FAO and SOK were responsible for designing the study and coordinated data collection. They also wrote and revised the manuscript. JS, DOL, EA-Y, AO-A, OAO, MT and PA-B designed the tools for data collection and delivered the training. As medical photographer, JB coordinated the production of visual material used in the training and was also involved in manuscript revision. NM, IO-P, AO, JOS, VA and SCYA gave input to the study design, handled data collection and prepared the data for analysis, and were responsible for participant contact. IN, EXA, AE and JD contributed to study design, analysed the data and contributed to manuscript preparation and revision. All authors approved the final manuscript and agree to be accountable for the work. Funding. The study was funded by the DANIDA Fellowship Centre, through the participation of the Kwame Nkrumah University of Science and Technology in the Building Stronger University initiative. The funding allowed for participants' compensation. The authors confirm that they had full access to all the data in the study and were responsible for deciding to submit the findings for publication.

Conflicts of interest. None.

1. Rudan I, O’Brien KL, Nair H, et al. Epidemiology and etiology of childhood pneumonia in 2010: Estimates of incidence, severe morbidity, mortality, underlying risk factors and causative pathogens for 192 countries. J Glob Health 2013;3(1):010401.

2. World Health Organization, UNICEF. Ending preventable child deaths from pneumonia and diarrhoea by 2025. The Integrated Global Action Plan for Pneumonia and Diarrhoea (GAPPD). Geneva: WHO, 2013.

3. Walker CLF, Rudan I, Liu L, et al. Global burden of childhood pneumonia and diarrhoea. Lancet 2013;381(9875):1405-1416. https://doi.org/10.1016/S01406736(13)60222-6

4. Rudan I, Boschi-Pinto C, Biloglav Z, Mulholland K, Campbell H. Epidemiology and etiology of childhood pneumonia. Bull World Health Organ 2008;86(5):408-416. https://doi.org/10.2471/BLT.07.048769

5. Liu L, Oza S, Hogan D, et al. Global, regional, and national causes of under-5 mortality in 2000-15: An updated systematic analysis with implications for the Sustainable Development Goals. Lancet 2016;388(10063):3027-3035. https:// doi.org/10.1016/S0140-6736(16)31593-8

6. Ministry of Health Ghana Health Services. MANUAL training health workers on pneumococcal and rotavirus vaccines. 2012.

7. Renner LA, Newman MJ, Ahadzie L, Antwi-Agyei KO, Eshetu M. Introduction of Haemophilus influenzae type B conjugate vaccine into routine immunization in Ghana and its impact on bacterial meningitis in children younger than five years. Pediatr Infect Dis J 2007;26(4):356-358. https://doi.org/10.1097/01. inf.0000258693.19247.8e. 
8. Källander K, Hildenwall H, Waiswa P, Galiwango E, Pariyo G. Delayed care seeking for fatal pneumonia in children aged under five years in Uganda: A case-series study. Bull World Health Organ 2008;86(5):332-338. https://doi. org/10.2471/BLT.07.049353

9. Källander K, Tomson G, Nsabagasani X, Sabiiti JN, Pariyo G, Peterson S. Can community health workers and caretakers recognise pneumonia in children? Experiences from western Uganda. Trans R Soc Trop Med Hyg 2006;100(10):956-963. https://doi.org/10.1016/j.trstmh.2005.11.004

10. Mishra S, Kumar H, Sharma D. How do mothers recognize and treat pneumonia at home? Indian Pediatr 1994;31(1):15-18

11. Schellenberg D, Menendez C, Kahigwa E, et al. African children with malaria in an area of intense Plasmodium falciparum transmission: Features on admission to the hospital and risk factors for death. Am J Trop Med Hyg 1999;61(3):431-438.

12. Ghana Statistical Service. 2010 Population and Housing Census. Ghana Statistical Service: Accra, 2012.

13. Memon KN, Shaikh K, Pandhiani BS, Usman G. How do mothers recognize \& treat pneumonia in their children at home? A study in Union Council Jhudo, District Mirpurkhas. Jnl Liquat Univ Med Health Sci 12(3):208-213.
14. World Health Organization. Pneumonia. Key facts, 2016. https://www.who.int/ news-room/fact-sheets/detail/pneumonia (accessed 8 July 2019).

15. Yeji F, Shibanuma A, Oduro A, et al. Continuum of care in a maternal, newborn and child health program in Ghana: Low completion rate and multiple obstacle factors. PLoS ONE 2015;10(12):e0142849. https://doi.org/10.1371/journal. pone. 0142849

16. Kikuchi K, Ansah EK, Okawa S, Enuameh Y. Effective linkages of continuum of care for improving neonatal, perinatal, and maternal mortality: A systematic review and meta-analysis. PLoS ONE 2015;10(9):e0139288. https://doi. org/10.1371/journal.pone.0139288

17. Kikuchi K, Ansah E, Okawa S, et al. Ghana’s Ensure Mothers and Babies Regular Access to Care (EMBRACE ) program: Study protocol for a cluster randomized controlled trial. Trials 2015;16:22. https://doi.org/10.1186/s13063-014-0539-3

Accepted 8 July 2019. 